\title{
An Algorithm of Image Contrast Enhancement Based on Pixels Neighborhood's Local Feature
}

\author{
Chen Yan*,1,2,3, Shao Yanling ${ }^{4}$ and Gui Zhiguo ${ }^{1,2,3}$ \\ ${ }^{1}$ National Key Laboratory for Electronic Measurement Technology,North University of China,Taiyuan 030051, China \\ ${ }^{2}$ Key Laboratory of Instrumentation Science \& Dynamic Measurement, North University of China,Taiyuan 030051, China \\ ${ }^{3}$ School of Information and Communication Engineering, North University of China,Taivuan 030051, China \\ ${ }^{4}$ School of science, North University of China, Taiyuan 030051, China
}

Received 10 October 2013; Accepted 14 December 2013

\begin{abstract}
In this study, we proposed an algorithm of Image Contrast enhancement based on local feature to acquire edge information of image, remove Ray Imaging noise and overcome edge blurry and other defects. This method can extract edge features and finish contrast enhancement in varying degrees for pixels neighborhood with different characteristics by using neighborhood local variance and complexity function, which can achieve local features enhancement. The stimulation shows that the method can not only enhance the contrast of the entire image, but also effectively preserves image edge information and improve image quality.
\end{abstract}

Keywords: Contrast Enhancement, Local Feature, Neighborhood

\section{Introduction}

During X-ray imaging process, the target images will become blurred and have poor quality due to the loss of partial image information and various factors, such as blurred edges, low contrast and so on. Therefore, it is essential to enhance the image to make the output image meet actual requirements. Image enhancement is a critical preprocess-ing step in image analysis and pattern recognition Contrast enhancement is one of the most important issues in image processings, and it can be classified into the indirect method and the direct method. Histogram equalization is well-known indirect methods.Histogram equalization can achieve contrast enhancement of the overall image through using cumulated function as its mapping function, which usually results in the loss of detailed information and the local blurry of output image because of excessive mergence of gray levels of pixels of which number is much less than others in the process. Linear and non-linear contrast stretching have limited enhancement for X-ray image, but they can't be utilized to enhance the details of object images. Image sharpening is an effective way to compensated image contours、 enhance the edge and abrupt change of gray level to make target image more legible. The purpose of sharpening is to enhance the edge、 contours line and details of image, and in brief its nature is to improve contrast of

* E-mail address: nucchenyan@126.com

ISSN: 1791-2377 (C) 2013 Kavala Institute of Technology. All rights reserved. details edge, which will help us observe clearly. H.D.Cheng proposed a contrast enhancement method based on similarity measure by taking the edge, gray level and entropy into consideration to reflect the local information of image. But this method needs large computation cost. Zuoyong Li employed pixel neighborhood complexity and meanvariance to extract the transition region from input image to reflect local features of pixels neighborhood and simplify the calculation of neighborhood characteristics.

In this paper, we improved the method presented by Zuoyong $\mathrm{Li}$ by combining pixels neighborhood characteristics and local contrast and discussed an enhancement algorithm for X-ray images. This method adequately utilized the local changes of image gray values and frequency features, combined local characteristics of pixel and contrast, which will simplify the calculation and effectively improve the contrast of X-ray images.

\section{Contrast Enhancement Methods}

The contrast of the edges and regions full of details will be improved and the visual effect of images will be better by modifying the dynamic range of gray image according to certain transformation rules. The basic idea of direct contrast enhancement methods is to establish a criterion of contrast measurement and to enhance the image by improving the contrast measurement. Usually, contrast refers to the difference in luminance between an object and its surrounding region. The contrast value is defined as: 
$C_{i j}=\frac{\left|f_{\mathrm{ij}}-A\right|}{f_{\mathrm{ij}}+A}$

$A=\frac{1}{8} \sum_{k=i-1}^{i+1} \sum_{l=j-1}^{j+1} f_{k l}$

Where $C_{i j}$ is the local contrast value of pixel $(i, j), f_{i j}$ is the gray level of the pixel and $\mathrm{A}$ is the background around this pixel. We use the contrast enhancement function to increase the local contrast value $C_{i j}$ to enhance the image, it can be formulated as:

$C_{\mathrm{ij}}^{\prime}=\varphi\left(C_{i j}\right)$

Where $\varphi$ usually meets the following requirements: (1) $\varphi\left(C_{i j}\right)>C_{\mathrm{ij}}$; (2) $\varphi\left(C_{i j}\right) \in[0,1]$. Then we can acquire the enhanced image, using the formula:

$f_{\mathrm{ij}}^{\prime}= \begin{cases}\lambda \frac{1+C_{\mathrm{ij}}^{\prime}}{1-C_{\mathrm{ij}}^{\prime}} & f_{\mathrm{ij}} \geq \lambda \\ \lambda \frac{1-C_{\mathrm{ij}}^{\prime}}{1+C_{\mathrm{ij}}^{\prime}} & f_{\mathrm{ij}}<\lambda\end{cases}$

The algorithm we mentioned above can be reached by using the equation(2) to calculate the neighbor pixels mean gray value of the pixel(i,j)as the background,which will result in the increase of noise while enhance the image contrast. In this paper, we modified the way $\lambda$ is usually defined by exploiting the local neighbor features of the pixel to enhance images.

\section{Pixel Neighborhood Features}

Transition region is located between object and background, and covers around the object,and composed of pixels having intermediate gray levels between that of object and of background.Human eye is insensitive to features present at the both extremes of pixel intensity, whereas sensitive to distinguish-ing features at the mid-range intensities. This suggests a focus on mid-region of a gray scale image. In the light of human visual perception, a preprocessing step called image transformation is suggested to simplify original images.

Pixels'gray levels in transition region usually change frequently and intensively, bringing about abundant information for transition region description. Gradient is a good measure for sudden gray level changes, but inapplicable in measuring frequent gray level changes. Local entropy also can depict frequency of gray level changes. However, its computational complexity is high, as the calculation of local entropy involves statistical analysis for the pixels' gray levels and computes each gray level's probability appeared in a neighborhood. To reduce the computational complexity, a simple form with similar effect, local complexity, is used to describe frequency of gray level changes.

\subsection{Local Complexity}

Local complexity can be used to describe variable frequency of image gray value through utilizing local statistical information, like the local entropy. The gray level in neighbor which size is $\mathrm{k}^{*} \mathrm{k}$ of the pixel $(\mathrm{i}, \mathrm{j})$ can be calculate by using the equation (1) and (2) Local complexity can be calculated as follow:

$\operatorname{Lc}(i, j)=C(\Omega)=\sum_{k=1}^{9} \operatorname{sgn}(k)$

$\operatorname{sgn}(\mathrm{k})= \begin{cases}1 & h(i, j) \neq 0 \\ 0 & h(i, j)=0\end{cases}$

Where $f(i, j)$ is the gray value of the pixel $(i, j)$. Then the local complexity of the image can be got and form the local complexity matrix of the image, and it is defined as:

$L c=\left[\begin{array}{cccc}L c(1,1) & L c(1,2) \cdots \cdots & L c(1, n) \\ L c(2,1) & L c(2,2) \cdots \cdots & L c(2, n) \\ \vdots & \vdots & & \vdots \\ L c(m, 1) & L c(m, 2) \cdots \cdots \cdots & L c(m, n)\end{array}\right]$

Where $L$ is the gray level of image.

\subsection{Local Variance}

Local variance of pixel can reflect the degree of variability of pixel gray value in local image widows by calculating the difference between the pixel and the neighboring pixels. Local variance between the pixel $(i, j)$ and pixels in the neighbor window which size is $\mathrm{k}^{*} \mathrm{k}$ can be calculated as follow:

$L v(i, j)=\sigma^{2}(\Omega)=\frac{1}{m \times n-1} \sum_{x=1}^{m} \sum_{y=1}^{n}(f(x, y)-\bar{f})^{2}$

Where $\bar{f}$ is mean of the gray levels within neighboring widows of size $\mathrm{k}^{*} \mathrm{k}$.

Similarly, the local variance matrix can be obtained by computing all variances between every pixel and its neighbor, it is formulated as following:

$L v=\left[\begin{array}{cccc}L v(1,1) & L v(1,2) \cdots \cdots & L v(1, n) \\ L v(2,1) & L v(2,2) \cdots \cdots & L v(2, n) \\ \vdots & \vdots & & \vdots \\ L v(m, 1) & L v(m, 2) \cdots \cdots & \operatorname{Lv}(m, n)\end{array}\right]$

The proposed method transformed the combination of the local complexity and local variance into a new operator to adequately describe the image characteristics at the edge. Normalization is performed by mapping the intensity levels of these two parameters into the same range $[0,1]$ to adjust different features'effect on image edges properly using the following equation:

$N L c(i, j)=\frac{L c(i, j)-\min L c(i, j)}{\max L c(i, j)-\min L c(i, j)}$
$N L v(i, j)=\frac{L v(i, j)-\min L v\left(i_{j}\right)}{\operatorname{maxL}(i, j)-\min L v(i, j)}$

\subsection{Neighborhood Feature Extraction}

The local complexity and local variance can describe characteristic variation of pixels in neighborhood from different aspects. A new operator is introduced to form the 
matrix $\mathrm{S}$ of the image by combining local complexity and local variance:

$$
S(i, j)=\alpha \times N L c(i, j)+(1-\alpha) \times N L v(i, j)
$$

Where $S$ takes the degree of variability of the local image gray value and frequency into consideration, and $\alpha$ is a parameter to regulate the balance between the local variance and complexity, when, $S$ only include the local variance of pixels, and while $\alpha=0, S$ can be defined by $L v$ matrix. Therefore, $\alpha$ will vary in the range [ $\left[\begin{array}{ll}0 & 1\end{array}\right]$.

The local complexity and local variance are related to the variation of the gray values, and their value will become large when the degree of variability and frequency increase. So, the value of $S$ at the edge will be larger than that in the non-edge region, and these regions with bigger $S$ value will be greatly enhanced.

\section{The Algorithm of Contrast Enhancement Based on Local Features}

(1) Calculate the local features matrix $S$ of neighboring pixels according to the equation (1) to (5);

(2) Compute the template coefficients of local features in neighboring windows of the pixel and it can be easily obtained as follow:

$\delta_{i j}=\frac{\sum_{m-t-1}^{t+1} \sum_{m-f-1}^{f+1}\left(g_{m+n} \times S_{m+n}\right)}{\sum_{m=1-1}^{t+1} \sum_{n-j-1}^{t+1} S_{m+n}}$

Where $\mathrm{d}$ is the size of neighboring window.

(3) Evaluate the local contrast related to the pixel $(i, j)$,

$C_{i j}=\frac{\left|g_{i j}-\delta_{i j}\right|}{g_{i j}+\delta_{i j}}$

(4) Transform the contrast $C_{i j}$ to $C_{i j}^{\prime}$,

$C_{\mathrm{ij}}^{\prime}=C_{\mathrm{ij}}^{\xi\left(s_{\mathrm{ij}}\right)}$

Where $\xi$ is the amplification constant, it is related to the local feature value $S$. If $S_{i j}$ is large, the difference of pixels in neighbor will increase and indicates that there are more details in this region, and $\xi$ should be high; On the contrary, $\xi$ should be low. The determination of $\xi$ can be defined as follow:

$$
\xi_{i j}=\xi_{i j}+\frac{\xi_{\max }-\xi_{\min }}{S_{\max }-S_{\min }} \times\left(S_{i j}-S_{\min }\right)
$$

Where $S_{\max }$ and $S_{\min }$ are the maximum and minimum consistency eigenvalues of neighboring pixels, respectively. And $\xi_{\max }$ and $\xi_{\min }$ are the the maximum and minimum amplification constant, respectively.

(5) Obtain the enhanced image gray value by the formula:

$$
g_{i j}^{\prime}=\left\{\begin{array}{lr}
\delta_{i j} \times \frac{1-C_{i j}^{\prime}}{1+C_{i j}^{\prime}} & g_{i j}<\delta_{i j} \\
\delta_{i j} \times \frac{1+C_{1 j}^{\prime}}{1-C_{1 j}^{\prime}} & \text { otherwise }
\end{array}\right.
$$

(6) Repeat steps 1-5 until acquire the enhanced image.

\subsection{Determining the amplification constant}

The determination of the amplification constant, should be related to the histogram of the given image.

$$
\text { Obtain the histogram } \mathrm{H}(\mathrm{g}) \text {. }
$$

Find the local maxima of the histogram $\operatorname{Hmax}(\mathrm{gi}) \ldots$

$\downarrow$

\section{Calculate the mean height of the local maxima}

$$
\overline{H_{\max }}=\frac{\sum_{i=1}^{k} H_{\max }\left(g_{k}\right)}{k}
$$

Find the peaks if their values are greater than $\overline{\mathrm{H}_{\max }}$

V

Select the firrst peak $P\left(g_{l}\right)$ and the last peak $P\left(g_{k}\right)$ of the histogram, and find the corresponding gray levels $g_{l}$ and $g_{k}$.

$$
\begin{aligned}
& \text { Calculate the minimal amplification constant } \\
& \xi_{\text {min }}=\frac{g_{k}-g_{1}}{g_{\max }-g_{1}}
\end{aligned}
$$

Fig. 1. The flowchart of determining the amplification constant

For the proposed algorithm, determining the amplification constant $\xi_{i j}$ is critical. From the Fig 1.we determined $\xi_{i j}$ according to the nature of the original image,so the determination of $\xi_{\mathrm{ij}}$ can be done automatically and adaptively.

\section{Experimental Analysis}

In order to test the effectiveness of the proposed algorithm, we selected X-ray images with low contrast as input images and compared this method with traditional histogram equalization., the original image is vague and blurred. We can see the contrast was enhanced in Fig.2(b)and 2(c).Comparing Figs.2(b) and (c), we can see that Fig. 2(c) is much clearer and more uniform,but there are some overenhanced portions. It is very clear that the image obtained by the proposed method is much better than the one using other method. In Fig.2 (d), The details of the image in Fig. 2(c) are uniform, natural and clear. The nature of image sharpening is to enhance the high-frequency components of the original image.

As we mentioned earlier, the old method only stretches the global distribution of the intensity, and the performance of this method for contrast enhancement is not effective and effcient. Fig. 4(a) is a low contrast, vague image. the main features in Fig. 4(b) are ell-enhanced with amplifying noise. The over-enhancement problem of fuzzy enhancement method exists in Fig. 4(c) . The image is non-uniform and unnatural.

The application of neighboring sharpening method would amplify high-frequency noise when enhanced the original 
image due to high-frequency components of the degraded image contains not only valid information but also random noise, which is shown as the appearance of obvious glitch noise after sharpening. The proposed method can produce the output image with more reasonable and uniform gray levels' distribution and preserve the gray values of small probability, what's more, it can also improve the image contrast while preserving the local details and make the enhanced image more natural.
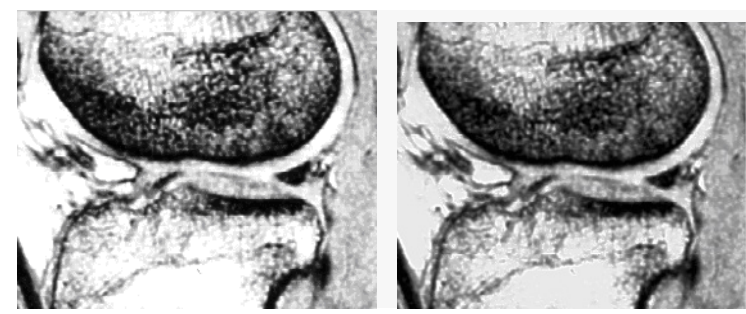

(a)

(b)

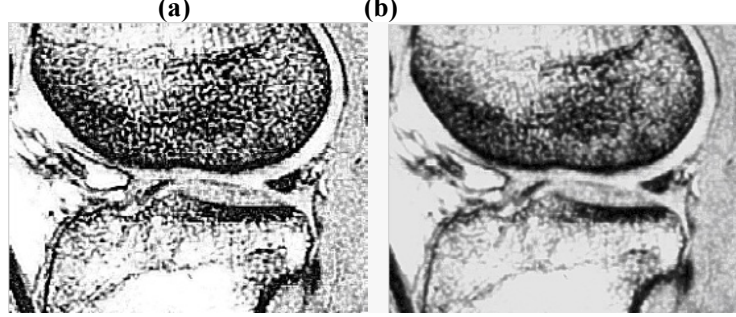

(c)

(d)

Fig. 2. The results of different algorithms

(a) the original image;

(b) The image enhanced by the HE;

(c) The image enhanced by the 8 neighborhood

(d) The image enhanced by the proposed method

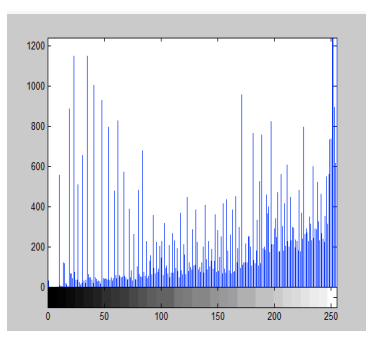

(a)

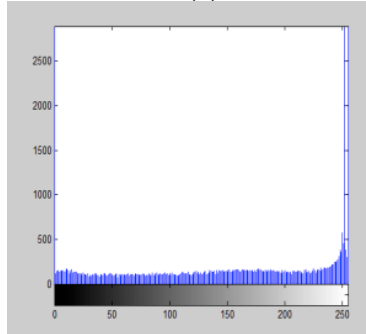

(c)

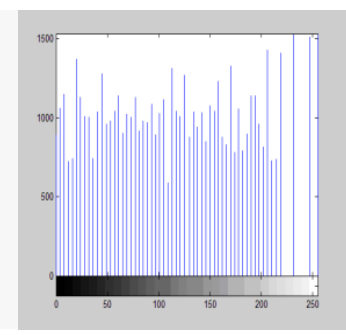

(b)

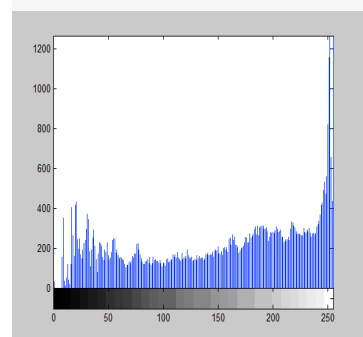

(d)
Fig. 3. The histograms of different algorithms

(a)The histogram of the original image;

(b)The histogram of HE;

(c)The histogram of 8 neighborhood;

(d)The histogram of the propose method

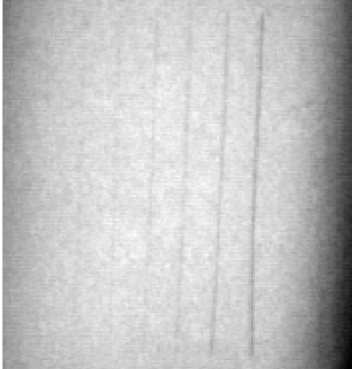

(a)

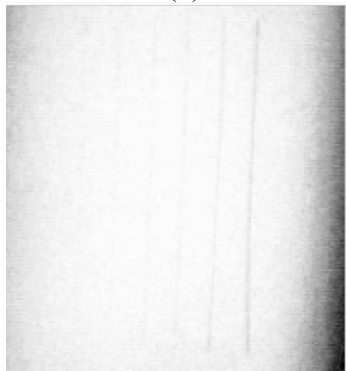

(b)

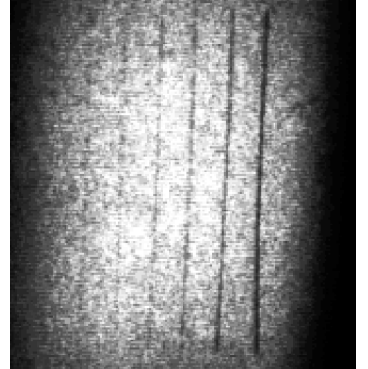

(b)

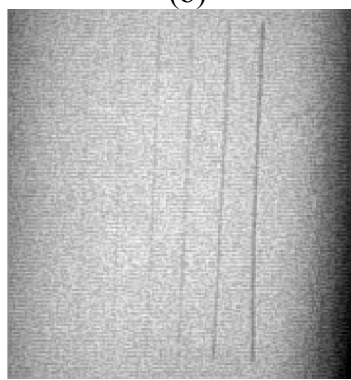

(d)
Fig. 4. The results of different algorithms
(a) the original image;
(b) The image enhanced by the HE;
(c) The image enhanced by thefuzzy enhancement;
(d) The image enhanced by the proposed method

Quality evaluation parameters can qualitatively describe the quality of image and judge objectively the effectiveness of the enhancement.The common quality evaluation parameters are the mean square error、entropy and peak SNR, they can be defined as:

(a) The mean square error of image:

$$
M S E=\frac{1}{\mathrm{mn}} \sum_{i=0}^{\mathrm{m}} \sum_{j=0}^{n}\|g(i, j)-f(i, j)\|^{2}
$$

Where $g(i, j)$ is the enhanced image, and $f(i, j)$ is the original image.m and $n$ represents the length and the width of image, respectively.

(b) The peak SNR of image reflects the statistical average of changes in image SNR, the smaller noises are, the larger the peak SNR would be.PSNR can be formulated as:

$P S N R=10 \log \left(\frac{M A X^{2}}{M S E}\right)=20 \log \left(\frac{M A X}{\sqrt[2]{M S E}}\right)$

Where MAX is the maximum gray value of the pixel in image.

(c) The entropy of image indicates the average information, when the entropy gets larger the enhanced image will preserve more details from the original image.The entropy of a two-dimensional gray-scale image can be defined as: 


$$
\mathrm{p}(\mathrm{k})=\frac{A_{K}}{M \times N}
$$

Where $\mathrm{p}(\mathrm{k})$ is gray density distribution of the enhanced image.

Table 1. Objective Quality Evaluation Parameters of different algorithms(Fig 1)

\begin{tabular}{c|c|c|c}
\hline & HE & 8 neighborhood & propose method \\
\hline MSE & 41.9164 & 52.6888 & 8.2612 \\
SNR & 12.7934 & 10.8067 & 26.9003 \\
Entropy & 3.9666 & 4.1997 & 0.13633 \\
\hline
\end{tabular}

Table 2. Objective Quality Evaluation Parameters of different

\begin{tabular}{c|c|c|c} 
& algorithms(Fig 2) \\
& HE & $\begin{array}{c}\text { Fuzzy } \\
\text { enhancement }\end{array}$ & propose method \\
\hline MSE & $\mathbf{6 7 . 1 3 2 5}$ & $\mathbf{1 2 2 . 7 0 6}$ & 1.8871 \\
SNR & $\mathbf{5 . 3 6 4 4}$ & $\mathbf{0 . 1 2 5 7 1}$ & $\mathbf{3 6 . 3 8 7 2}$ \\
Entropy & $\mathbf{7 . 8 7 2 1}$ & $\mathbf{3 . 3 5 6 2}$ & $\mathbf{1 . 0 8 7 9}$ \\
\hline
\end{tabular}

Mean square error and PSNR are usually used as tools to compare parameters that evaluate the quality of the output image and the original image. The smaller the value of mean square error is, the greater PSNR will be, which means there are less noises in image. The greater the entropy of the image is, the more information the image contains, which indicates the image has better quality.Table 1 lists some quality evaluation parameters of the proposed algorithm and several other methods.As can be seen from Table 1, the proposed method has the minimum mean square error and the maximal PSNR and entropy when compared with other algorithms, which implies that the method presented in our work can enhance the details of image while suppressing noises.

\section{Conclusions}

Contrast enhancement is one of the most important issues in many image areas. In this paper, we proposed a novel adaptive contrast enhancement algorithm by Combining the local neighboring information and contrast.Our proposed method defines the contrast of image based on Pixels's Local Feature.The experimental results have demonstrated that the proposed method has better performance than the tradition method. The good performances are due to the following factors: The proposed method uses both local and global information to compute the components of neighborhood and makes the contrast enhancement more adaptive and effective.This method is very good at suppressing the over-enhancement, especially suppressing over-enhancement of noises, and preserving much more image details.Experimental results show that this proposed technique can produce clear enhanced image and make it easier to observe the result, which is very helpful to further research on image processing.

\section{References}

1. H.D. Cheng, H.J. Xu, A novel fuzzy logic approach to contrast enhancement, Pattern Recognition 33 (2000) 809-819.

2. Cheng H D,Mei X,Shi X J.Contrast enhancement based on a novel homogeneity measurement.Pattern Recognition,2003,36(11):26872697.

3. Zuoyong Li,David Zhang, Yong Xuc,Chuancai Liu.Modified local entropy-based transition region extraction and thresholding. Applied Soft Computing,2011(11) 5630-5638

4. H. Zhu, F.H.Y. Chan, F.K. Lam, Image contrast enhancement by constrained local histogram equalization, Comput Vision Image Understanding 73(2)(1999) 281-290.

5. Y. Qiao, Q.M. Hu, G.Y. Qian, S.H. Luo, W.L. Nowinski, Thresholding based on variance and intensity contrast, Pattern Recognit. 40 (2) (2007) 596-608.

6. Effect of administered radioactive dose level on image quality of brain perfusion imaging with 99mTc-HMPAO,Journal of Engineering Science and Technology Review 1(2008) 66-69

7. R.C. Gonzalez, R.E. Woods, Digital Image Processing, 2nd Edition, Prentice-Hall, Englewood Cli2s, NJ, 2002.
8. E.L. Hall, Computer Image Processing and Recognition,Academic Press, New York, 1979. 2.

9. H.D. Cheng, H.J. Xu, A novel fuzzy logic approach to contrast enhancement, Pattern Recognition 33 (2000) 809-819.

10. Implementation of Shape - Based Matching Vision System in Flexible Manufacturing System, Journal of Engineering Science and Technology Review, 3 (2010) 128-135.

11. C.X. Yan, N. Sang, T.X. Zhang, Local entropy-based transition region extraction and thresholding, Pattern Recognit.Lett. 24 (16) (2003) 2935-2941.

12. C. Zhang, J. Zhang, H. Chen, Local fuzzy entropy-based transition region extraction and thresholding, Int. J. Inf. Technol. 12 (6) (2006) 19-25

13. L. Dash, B.N. Chatterji, Adaptive contrast enhancement and deenhancement, Pattern Recognition 24 (1991) 289-302.

14. Z. Li, J. Yang, G. Liu, Y. Cheng, C. Liu, Unsupervised rangeconstrained thresholding, Pattern Recognit. Lett. 32 (2) (2011) 392402 . 